Harold A. McAlister and William I. Harkopf (eds.)

\title{
The Use of Pulsating Stars in Orbit Determination
}

\author{
ANDREJ PIGULSKI \\ Wrocław University Observatory, ul. Kopernika 11, \\ 51-622 Wrocław, Poland
}

In a double or a multiple system, in which one of the components is a pulsating star, the light-time effect leads to apparent changes of the pulsation period. In some cases, one can obtain much more accurate values of the spectroscopic elements from the fit to the $O-C$ diagram than from classical analysis of the radial-velocity data. In addition, in the analysis of the $O-C$ diagram, photometric observations can be used. They are easy to obtain, and therefore are usually more numerous than the spectrographic ones.

Below we present the results of a study of three stellar systems, in which the light-time effect is observed. Primaries in all these systems are $\beta$ Cephei-type pulsating variables.

$\beta$ Cep: The apparent changes of the pulsation period seen in Figure 1a can be completely explained by the light-time effect, induced by the speckle companion (Pigulski, A. \& Boratyn, D.A. 1992, $A \mathscr{E} A, \mathbf{2 5 3}, 178$ ). Using the spectroscopic elements derived from the fit to the $O-C$ diagram, we were able to determine the visual orbit, although the speckle observations cover only about $20 \%$ of the orbital period (Figure 1b).

$\sigma$ Sco: The light-time effect caused by the speckle companion is large (Figure 2 ), but the orbital period is longer than the interval covered by the observations. Therefore, the orbital elements cannot be derived at present (Pigulski, A. 1992, $A \mathscr{E} A$, in press).

BW Vul: The original $O-C$ diagram for this star can be approximated by a broken line. This can be explained as a superposition of the evolutionary increase of period with a constant rate equal to $2.34 \mathrm{~s} /$ cen and a periodic term caused by the light-time effect (Figure 3 ). The fact that no companion has so far been detected by speckle interferometry may be due to expected large magnitude difference $(6-9 \mathrm{mag})$ and very small separation $\left(<0^{\prime \prime} .03\right)$ between the primary and its hypothetical companion.

The study of the $O-C$ diagrams, although limited to the systems with a pulsating primary, can give accurate values of the spectroscopic elements. Moreover, for some systems this method is the only one which can reveal the presence of companion(s). The case of BW Vul indicates that the light-time effect, sometimes combined with an effect of evolutionary origin, may be responsible for at least some of the "unexpected" or "sudden" period changes, reported for many stars of various types.

I would like to acknowledge the financial support of the Stefan Batory Foundation and the International Astronomical Union, which allowed me to attend the meeting. 

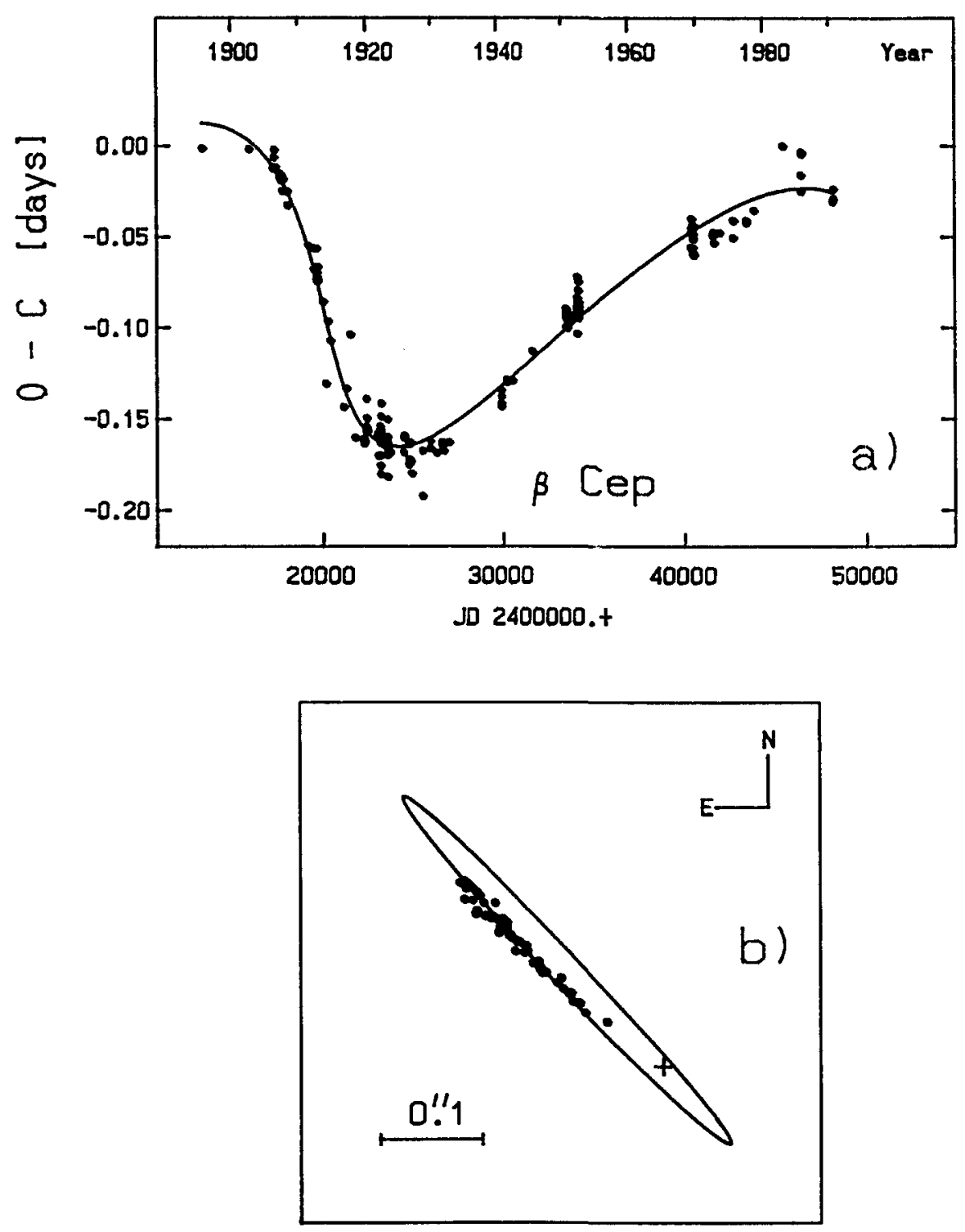

FIGURE 1. The $O-C$ diagram for the pulsating primary of the $\beta$ Cep system (upper), and the relative orbit of the secondary (lower). Spectroscopic elements were obtained from the best fit to the $O-C$ diagram (continuous line in the upper figure). The orbit shown in the lower figure was derived from the fit to the speckle interferometric observations, but with orbital period, eccentricity and time of periastron passage taken from the spectroscopic solution. 


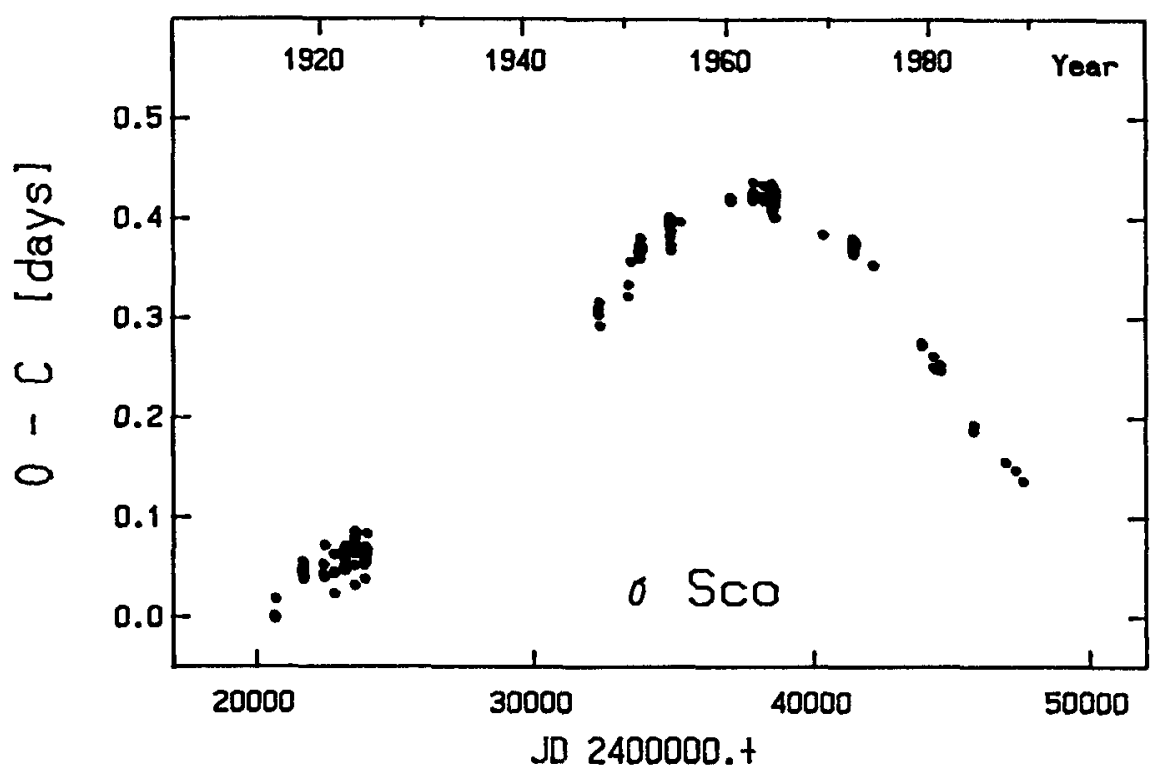

FIGURE 2. The $O-C$ diagram for $\sigma$ Sco after removing the evolutionary increase of period equal to $3.3 \mathrm{~s} / \mathrm{cen}$. The changes seen in this figure are due to the light-time effect caused by the speckle secondary.

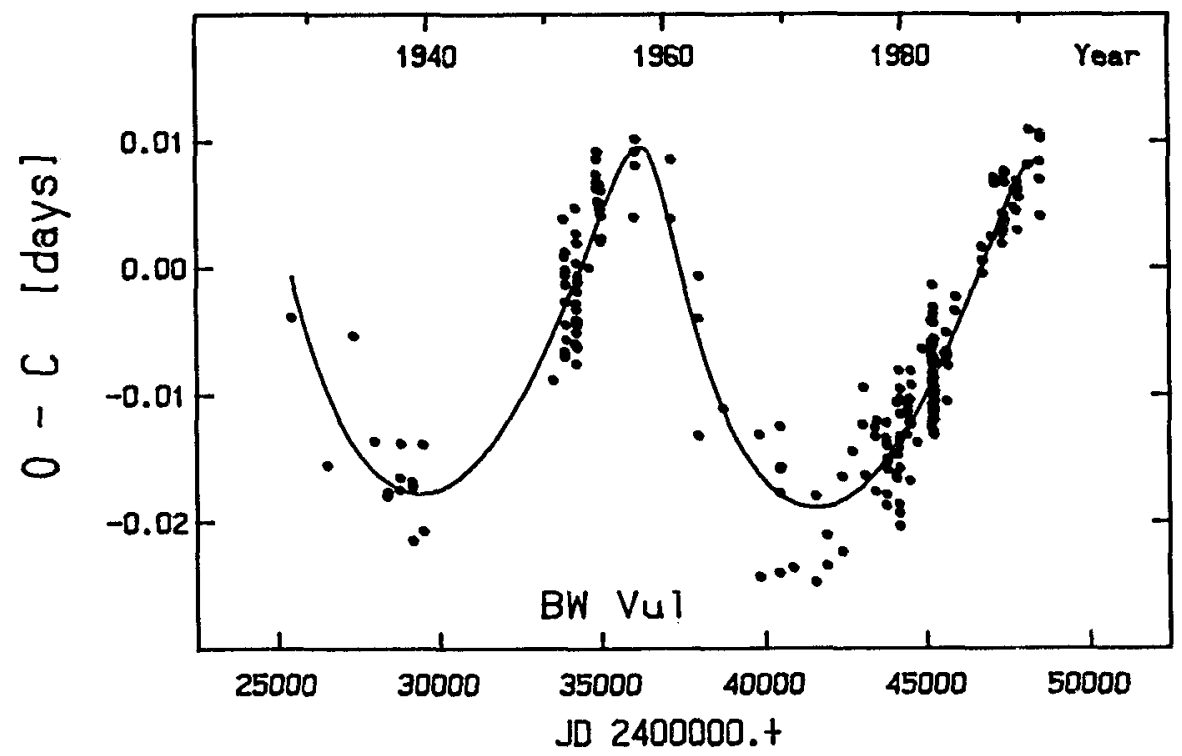

FIGURE 3. The $O-C$ diagram for BW Vul after removing the evolutionary increase of period equal to $2.34 \mathrm{~s} / \mathrm{cen}$. As in the case of $\beta$ Cep it was possible to obtain the spectroscopic elements from the $O-C$ diagram (continuous line). In this system the changes seen in the above diagram are the only manifestation of the presence of a companion. 